\title{
Detection and analysis of 18 PAHs in water by micro-LLE combined with concurrent solvent recondensation-large volume injection-GC-MS
}

\author{
Xiaolin Zhou ${ }^{1, \mathrm{a}}$, Bo Xu ${ }^{1, \mathrm{~b}}$, Liya Cao ${ }^{2, \mathrm{c}}$, Xinying Zhang ${ }^{\text {,2,d }}$
}

${ }^{1}$ CNOOC central laboratory, CNOOC EnerTech-Drilling \& Production Co., Shanghai 200941, China;

${ }^{2}$ College of environment and chemical engineering, Shanghai University, Shanghai 200444, China.

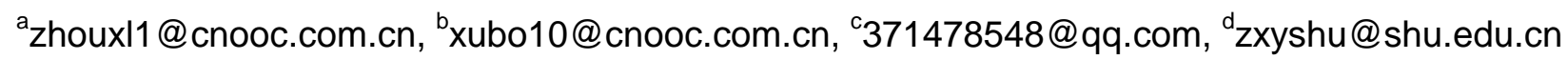

\begin{abstract}
Keywords: Detection, 18 PAHs, micro-LLE, GC-MS, recondensation-large volume injection
Abstract. A rapid and efficient method termed micro liquid-liquid extraction combined with concurrent solvent recondensation-large volume injection-GC-MS has been developed for the extraction and determination of PAHs in water. Some condition parameters were optimized to obtain high detection sensitivity. Calibration curves of $18 \mathrm{PAHs}$ were linear in the range of $0.1-10 \mathrm{ng} \cdot \mathrm{L}^{-1}$, with the correlation coefficients (r2) between 0.9993 and 1.0. The method attained good precisions from $0.3 \%$ to $4.5 \%$ for PAHs standard solutions. The method recoveries ranged between $64.5-119.5 \%$ for spiked with $5.0 \mathrm{ng} \cdot \mathrm{L}^{-1}$ and 76.2-119.5\% for spiked with $25.0 \mathrm{ng} \cdot \mathrm{L}^{-1}$ and $50.0 \mathrm{ng} \cdot \mathrm{L}^{-1}$ for real water samples. Limits of detection (LODs, $\mathrm{S} / \mathrm{N}=3$ ) of the method were determined from $0.010-0.068 \mathrm{ng} \cdot \mathrm{L}^{-1}$. The optimized method was successfully applied to the determination of 18 PAHs in real water samples.
\end{abstract}

\section{Introduction}

Polycyclic aromatic hydrocarbons (PAHs) have been listed as carcinogenic and mutagenic priority pollutants, belonging to its carcinogenic, teratogenic and mutagenic[1]. But PAHs is difficult to quantify in water environment because of the low concentrations. A series of different pre-treatment technologies has been developed to extraction and concentration of PAHs from water samples, such as liquid-liquid extraction (LLE)[2], liquid-liquid microextraction (LLME)[3], solid-phase extraction (SPE)[4], stirring bar sorptive extraction (SBSE)[5], solid-phase micro-extraction (SPME)[6] etc. Among them, liquid-liquid extraction and liquid-liquid microextraction are the most widely used extraction technologies. Although LLME has been demonstrated to be one of the most promising and cost-effective technologies, due to the lower solvent consumption and shorter extraction time and higher extraction efficiency[7]. However, in real application the very lower amount of solvent used in LLME makes the aspiration of extraction solvent difficult to handle. Nowadays, a possible approach to solving this problem is to use large volume injection (LVI) techniques, which can inject large volumes up to dozens to hundreds of $\mu \mathrm{L}$ of sample and shows high detection sensitivity. Based on these, we can adopt micro liquid-liquid extraction to extract PAHs in water, in which the solvent amount used is larger than in LLME and lower than in conventional LLE. Generally, the mostly used LVI in gas chromatography are on-column (OC-LVI) and programmed temperature vaporisation (PTV-LVI)[8]. But the lower ruggedness for dirty sample of OC-LVI and volatile PAHs may losses during solvent split process for PTV-LVI, which makes them not suitable for simultaneously analysis of PAHs containing low boiling points and high boiling points in water environment[9]. Encouragingly, Concurrent solvent recondensation (CSR) large volume injection technique which based on conventional split/splitless injector allows the injection amount up to $50 \mu \mathrm{L}$ dependents on the pre-column installed. It has no discrimination for low boiling points and high boiling points compounds, and also shows some ruggedness for dirty samples[10]. In recent years, CSR-LVI has been successfully applied for organic matter analysis, such as linear and cyclic methylsiloxanes in sewage sludges and urban soils[11], PAHs in diesel fuel[12], and pesticides residues in fruit and vegetables[13]. Besides, LVI-GC-MS for the analysis of PAHs in aqueous samples also was successfully developed[14]. However, up to now, no applications for determine PAHs in water by similar LLME combined with CSR-LVI GC-MS has been reported. 
In this study, we established a method for determination of PAHs in water based on similar LLME combined with CSR-LVI GC-MS system, and make a systematic investigation from linearity curve, precisions, recoveries and limits of detection, respectively. The method was demonstrated to be applicable for the analysis of 18 PAHs in real environmental water samples.

\section{Experimental results and discussion}

\section{Optimization of CSR-LVI GC-MS condition for PAHs}

The large volume syringe aspirate large volume sample and fast injected into liner with liquid band formation. The sample is received by a small packing of deactivated glass wool positioned slightly above the column entrance at the bottom of the splitless liner. Solvent evaporation strongly increases the pressure in the injector, will driving the first vapors into the precolumn and recondensation of the solvent with the oven temperature below the pressure-corrected solvent boiling point[10]. In which, the syringe and the pre-column will be different according to the different injection volume. In present work, we intend to inject more samples $(50 \mu \mathrm{L})$ to improve the detection sensitivity, so a $100 \mu \mathrm{L}$ syringe and a pre-column of $5 \mathrm{~m} \times 0.53 \mathrm{~mm}$ I.D., were used. The detailed operative parameters were selected according to splitless large volume assistant software (ThermoFisher)[12]. Typically, for injection $50 \mu \mathrm{L}$ PAHs samples with cyclohexane $(\mathrm{CHX})$ as solvent, the injector temperature was set at $280{ }^{\circ} \mathrm{C}$, using an initial oven temperature of $80{ }^{\circ} \mathrm{C}$ for $9 \mathrm{~min}$ and a splitless time of $8 \mathrm{~min}$. Injection 50 $\mu \mathrm{L}$ with $18 \mathrm{PAHs}$ concentration of $1 \mathrm{ng} \cdot \mathrm{mL}^{-1}$, all compounds shows high response.

\section{Optimization of extraction solvent and solvent amount}

Organic solvents are selected on the basis of extraction capability of interested compounds and good gas chromatography behavior[3]. Dichloromethane(DCM), hexane and CHX were selected and compared in the extraction of PAHs in water. A series of distilled water with 18 PAHs at a constant concentration of $0.1 \mu \mathrm{g} \cdot \mathrm{L}^{-1}$ were added, and $1.0 \mathrm{~mL}$ extraction solvent of DCM, hexane and CHX were used respectively. Then vortex mixing at $2500 \mathrm{rpm}$ for $30 \mathrm{~s}$, a cloudy solution (water, CHX) was formed in a glass bottle. After centrifuged for $1.0 \mathrm{~min}$ at $6000 \mathrm{rpm}$, the floated organic solvent was collected and analyzed by CSR-GC-MS. The recoveries for different extraction solvents were shown in Fig. 1. From the results, it can be clearly seen that CHX and DCM have almost the same extract recovery, and both are better than hexane as extract solvent, which is due to the high solubility of $\mathrm{CHX}$ and dichoromethane for PAHs. Considering the toxicity of DCM, the CHX was selected as extract solvent in the experiments. To examine the effect of extraction solvent volume, different amount of $\mathrm{CHX}$ was tested the extraction recoveries for distilled water with PAHs at a constant concentration of $0.1 \mu \mathrm{g} \cdot \mathrm{L}^{-1}$. From the real application view, the extraction solvent amount was optimized from $0.5 \mathrm{~mL}$ to $2.0 \mathrm{~mL}$ with $0.5 \mathrm{~mL}$ increased each. Fig. 2 shows the recoveries and enrichment factor versus of extract solvent amount for PAHs.

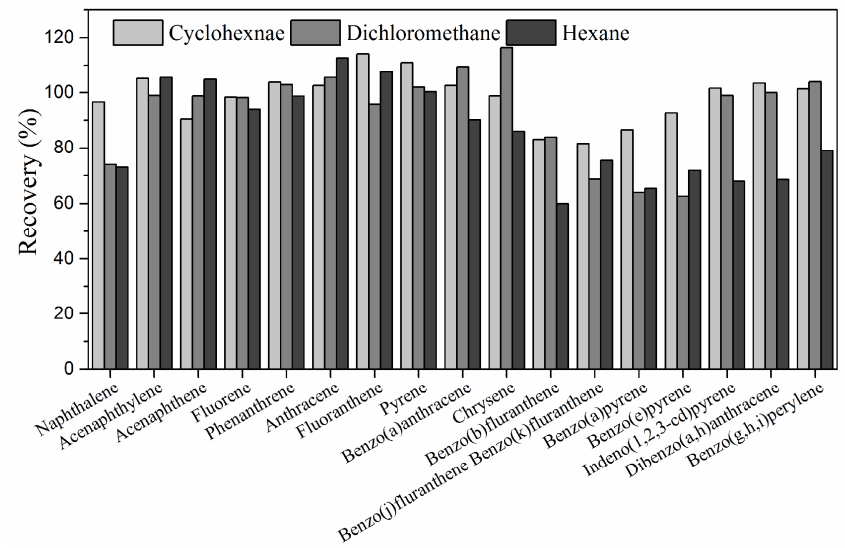

Fig. 1 The effect of extraction solvent kinds

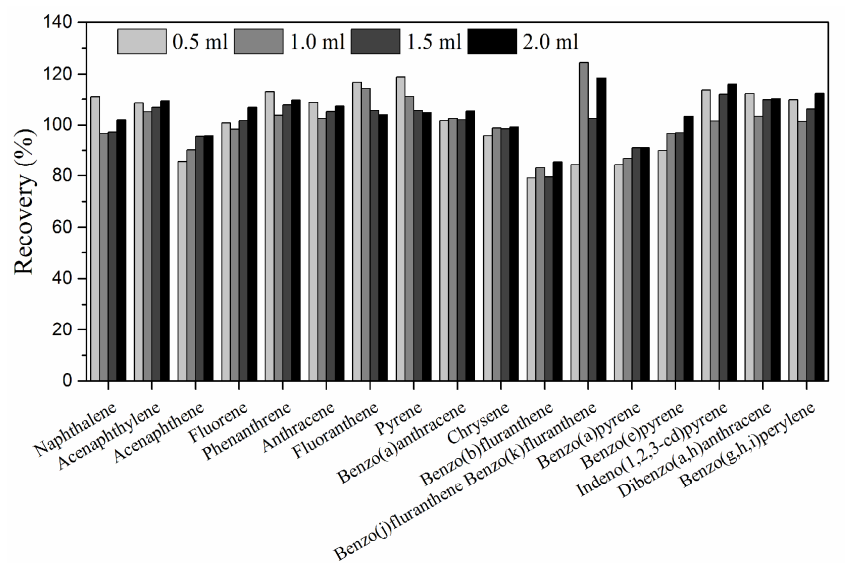

Fig.2 The effect of extraction solvent amount 


\section{Quantitative analysis}

The characteristic of calibration curves shown in Table 1 was obtained under optimized conditions. Linearity of calibration curve was observed at the range of $0.1-10.0 \mu \mathrm{g} \cdot \mathrm{L}^{-1}$ (two order magnitude) for all of the analytes. Coefficient of correlation (r2) ranged from 0.9992 to 1.0. Under the optimized conditions, the enrichment factors for PAHs were 40 , combined with the $50 \mu \mathrm{L}$ injection, the real enrichments factors were almost 2000, which makes its high detection sensitivity for PAHs.

Table 1 Analytical performance obtained for the 18 PAHs using CSR-GC-MS method

\begin{tabular}{cccccc}
\hline Compounds & $\mathrm{t}_{\mathrm{R}} / \mathrm{min}$ & $\begin{array}{c}\text { Linear ranges } \\
\left(\mathrm{ng} \cdot \mathrm{mL}^{-1}\right)\end{array}$ & $\mathrm{R}^{2}$ & $\begin{array}{c}\mathrm{MDL} \\
\left(\mathrm{ng} \cdot \mathrm{L}^{-1}\right)\end{array}$ & $\begin{array}{c}\mathrm{RSD} \\
(\%, \mathrm{n}=8)\end{array}$ \\
\hline Naphthalene & 13.59 & $0.1-10$ & 0.9992 & 0.010 & 1.3 \\
Acenaphthylene & 15.87 & $0.1-10$ & 0.9999 & 0.013 & 0.8 \\
Acenaphthene & 16.13 & $0.1-10$ & 0.9998 & 0.015 & 1.6 \\
Fluorene & 16.82 & $0.1-10$ & 0.9999 & 0.011 & 0.8 \\
Phenanthrene & 18.44 & $0.1-10$ & 0.9992 & 0.012 & 0.3 \\
Anthracene & 18.52 & $0.1-10$ & 1.0000 & 0.022 & 0.6 \\
Fluoranthene & 21.4 & $0.1-10$ & 0.9993 & 0.015 & 0.6 \\
Pyrene & 22.08 & $0.1-10$ & 0.9997 & 0.014 & 0.8 \\
Benzo(a)anthracene & 26.71 & $0.1-10$ & 0.9997 & 0.017 & 0.5 \\
Chrysene & 26.89 & $0.1-10$ & 0.9997 & 0.017 & 0.6 \\
Benzo(b)fluranthene & 32.17 & $0.1-10$ & 0.9992 & 0.042 & 1.9 \\
Benzo(j)fluranthene & 32.3 & $0.1-10$ & 0.9999 & 0.039 & 2.7 \\
Benzo(k)fluranthene & 33.61 & $0.1-10$ & 1.0000 & 0.043 & 2.0 \\
Benzo(a)pyrene & 33.87 & $0.1-10$ & 0.9998 & 0.062 & 2.2 \\
Benzo(e)pyrene & 40.33 & $0.1-10$ & 0.9998 & 0.058 & 4.5 \\
Indeno(1,2,3-cd)pyrene & 40.64 & $0.1-10$ & 0.9998 & 0.061 & 4.0 \\
Dibenzo(a,h)anthracene & 41.67 & $0.1-10$ & 0.9999 & 0.068 & 4.1 \\
Benzo(g,h,i)perylene & 4.67
\end{tabular}

${ }^{\mathrm{a}}$ Regression coefficient. b MDL: method detection limit. c RSD: relative standard deviation

Based on these, the reproducibility in peak responses was investigated on eight replicate experiments, the relative standard deviations (RSDs \%) of PAHs were from 0.3 to $4.5 \%$. And the method detection limit (LODs) was calculated based on signal-to-noise ratio $(\mathrm{S} / \mathrm{N})$ of 3 ranged from 0.010 to $0.068 \mathrm{ng} \cdot \mathrm{L}^{-1}$. Meanwhile, In order to further evaluate the performance of this method, a recovery study was carried out. Thus, aliquots of $20 \mathrm{~mL}$ of distilled water were spiked with $5.0 \mathrm{ng} \cdot \mathrm{L}^{-1}$, $25.0 \mathrm{ng} \cdot \mathrm{L}^{-1}$ and $50.0 \mathrm{ng} \cdot \mathrm{L}^{-1}$ of each compound, respectively. And extracted the samples use the above sample preparation procedures. Each sample was analyzed by quintuplicate; the results obtained are listed in Table 2. As can be seen, acceptable recovery values ranged between $64.5-119.5 \%$ for spiked with $5.0 \mathrm{ng} \cdot \mathrm{L}^{-1}$ and $76.2-119.5 \%$ for spiked with $25.0 \mathrm{ng} \cdot \mathrm{L}^{-1}$ and $50.0 \mathrm{ng} \cdot \mathrm{L}^{-1}$. From all of the above results, it can be concluded that this method shows high detection sensitivity and fulfill the PAHs detection in water environment.

Table 2 Recovery of proposed method for the determination of PAHs at three concentration levels

\begin{tabular}{|c|c|c|c|c|c|c|c|c|c|}
\hline \multirow{2}{*}{ Compounds } & \multirow{2}{*}{$\mathrm{t}_{\mathrm{R}} / \mathrm{min}$} & \multicolumn{3}{|c|}{ Recovery $(\%)$} & \multirow{2}{*}{ Compounds } & \multirow{2}{*}{$t_{R} / \min$} & \multicolumn{3}{|c|}{ Recovery $(\%)$} \\
\hline & & $0.005^{*}$ & $0.025^{*}$ & $0.05^{*}$ & & & $0.005^{*}$ & $0.025^{*}$ & $0.05^{*}$ \\
\hline Naphthalene & 13.59 & 68.0 & 113.5 & 101.8 & Chrysene & 26.89 & 93.5 & 107.2 & 108.6 \\
\hline Acenaphthylene & 15.87 & 69.0 & 102.1 & 103.4 & Benzo(b)fluranthene & 32.17 & 76.0 & 76.2 & 87.2 \\
\hline Acenaphthene & 16.13 & 64.5 & 96.7 & 95.3 & $\begin{array}{l}\text { Benzo(j)fluranthene } \\
\text { Benzo(k)fluranthene }\end{array}$ & 32.30 & 75.5 & 96.1 & 96.5 \\
\hline Fluorene & 16.82 & 70.5 & 103.0 & 101.8 & Benzo(a)pyrene & 33.61 & 87.5 & 88.5 & 92.25 \\
\hline Phenanthrene & 18.44 & 63.5 & 106.9 & 105.3 & Benzo(e)pyrene & 33.87 & 85.2 & 93.4 & 97.3 \\
\hline Anthracene & 18.52 & 83.5 & 111.5 & 113.6 & Indeno(1,2,3-cd)pyrene & 40.33 & 119.5 & 111.9 & 114.1 \\
\hline Fluoranthene & 21.40 & 86.0 & 113.2 & 115.1 & Dibenzo(a,h)anthracene & 40.64 & 115.5 & 118.9 & 118.8 \\
\hline Pyrene & 22.08 & 90.1 & 119.1 & 119.5 & $\operatorname{Benzo}(\mathrm{g}, \mathrm{h}, \mathrm{i})$ perylene & 41.67 & 115.5 & 109.8 & 113.6 \\
\hline Benzo(a)anthracene & 26.71 & 95.1 & 109.5 & 111.7 & & & & & \\
\hline
\end{tabular}

*Add amount, $\mu \mathrm{g} \cdot \mathrm{L}^{-1}$ 


\section{Real sample analysis}

To further evaluate the method applicability, the proposed extraction method was applied to the determination of PAHs in a variety of environmental water samples, namely: tap water, lake and river water. The results are summarized in Table 3, no PAHs were found in tap water, while Naphthalene, Acenaphthylene, Acenaphthene, Fluorene, Phenanthrene, Anthracene, Fluoranthene, Pyrene, Benzo(a)anthracene and Chrysene were determined in huangpu river and Naphthalene, Acenaphthylene, Fluorene, Phenanthrene and Fluoranthene were determined in lake water, respectively. In which, the high amount of PAHs in huangpu river, maybe attribute to the accumulation of emissions from ships and boats.

Table 3 Relative recoveries and standard deviation of PAHs from spiked surface water

\begin{tabular}{|c|c|c|c|c|c|c|c|}
\hline Compounds & $\mathrm{a}$ & $\mathrm{b}$ & $\mathrm{c}$ & Compounds & $\mathrm{a}$ & $\mathrm{b}$ & $\mathrm{c}$ \\
\hline Naphthalene & ND & 58.22 & 19.78 & Chrysene & ND & 6.02 & ND \\
\hline Acenaphthylene & ND & 3.22 & 2.75 & Benzo(b)fluranthene & ND & ND & ND \\
\hline Acenaphthene & ND & 5.20 & ND & $\begin{array}{l}\text { Benzo(j)fluranthene } \\
\text { Benzo(k)fluranthene }\end{array}$ & ND & ND & ND \\
\hline Fluorene & ND & 13.40 & 3.65 & Benzo(a)pyrene & ND & ND & ND \\
\hline Phenanthrene & ND & 40.82 & 10.25 & Benzo(e)pyrene & ND & ND & ND \\
\hline Anthracene & ND & 3.85 & ND & Indeno(1,2,3-cd)pyrene & ND & ND & ND \\
\hline Fluoranthene & ND & 40.35 & 3.60 & Dibenzo(a,h)anthracene & ND & ND & ND \\
\hline Pyrene & ND & 20.18 & ND & $\operatorname{Benzo}(\mathrm{g}, \mathrm{h}, \mathrm{i})$ perylene & ND & ND & ND \\
\hline Benzo(a)anthracene & ND & 3.50 & ND & & & & \\
\hline
\end{tabular}

a- Tap water, b- HuangPu River water, c- Lake water, $n g \cdot \mathrm{L}^{-1}$

\section{Conclusions}

A CSR-LVI-GC-MS method has been developed for the determination of PAHs in water samples. The combination of micro liquid-liquid extraction with concurrent solvent recondensation-large volume injection GC-MS enabled selective and sensitive analysis of PAHs at very low levels in complex water environment, and easy identification of the individual pollutants. The method was demonstrated to be greatly applicable for the routine analysis of the PAHs in water environments.

\section{Acknowledgements}

The work was funded by the National Natural Science Foundation of China (No.41373097), and Program for Innovative Research Team in University (No.IRT13078).

\section{References}

[1] P. Plaza-Bolanos, A.G. Frenich, J.L.M. Vidal: J. Chromatogr. A. Vol. 1217 (2010), p. 6303

[2] A. Filipkowska, L. Lubecki, G. Kowalewska: Anal. Chim. Acta. Vol. 547 (2005), p. 243

[3] A. Saleh, Y. Yamini, M. Faraji: J. Chromatogr. A. Vol. 1216 (2009), p. 6673

[4] J.P. Ma, R.H. Xiao, J.H. Li: J. Chromatogr. A. Vol. 1217 (2010), p. 5462

[5] M. B-Deschamps, J.J. Daudin, E. Barriuso: J. Chromatogr. A. Vol. 1167 (2007), p.143

[6] D. Djozan, Y. Assadi, H.S. Hosseinzadeh: Anal. Chem. Vol. 73 (2001), p.4054

[7] O. A-Aziz, A.M. El Kosasy, S.: J. Fluoresc. Vol. 24 (2014), p.787

[8] K. Banerjee, R.H. Savant, S. Dasgupta: J. AOAC Int. Vol. 93 (2010), p. 368

[9] F.M. Norlock, J. Jang, Q. Zou: J. Air Waste Manage. Assoc. Vol. 52 (2002), p. 19

[10] P. Magni and T. Porzane: J. Sep. Sci. Vol. 26 (2003), p. 1491

[11] E.Y. C-Damas, F.J. Santos, M.T. Galceran: J. Chromatogr. A. Vol. 1268 (2012), p. 150

[12] C. Daniela, M. Paolo, Z. Giacinto: J. Chromatogr. A. Vol. 1019 (2003), p. 211

[13] W. Stanisław: J. Chromatogr. A. Vol. 1222 (2012), p. 98

[14] A. Álvaro, M.T. Rosa, V. Ana: Talanta. Vol. 139 (2015), p. 1 